\title{
PROTOZOÁRIOS RUMINAIS DE NOVILHOS DE CORTE CRIADOS EM PASTAGEM TROPICAL DURANTE O PERÍODO SECO
}

\author{
Kellerson Luiz daSilva ${ }^{1}$, Eduardo Robson Duarte ${ }^{2 *}$, Claudio Eduardo Silva Freitas ${ }^{3}$, \\ Flávia OliveIRA ABRÃO ${ }^{4}$, LUCIANA CASTRO GERASEEV ${ }^{2}$ \\ ${ }^{1}$ Pós-Graduando em Ciências Veterinárias, Universidade Federal de Lavras, MG Brasil \\ ${ }^{2}$ Professores da Universidade Federal de Minas Gerais, MG Brasil duartevet@ hotmail.com \\ ${ }^{3}$ Instituto de Ciências Agrárias da Universidade Federal de Minas Gerais, MG, Brasil \\ ${ }^{4}$ Professora do IF Goiano e Pós-Graduanda em Zootecnia, Universidade Federal de Minas Gerais, MG Brasil
}

\begin{abstract}
Protozoários do rúmen podem representar $40 \%$ do nitrogênio total e $60 \%$ do produto final da fermentação e apresentam atividade celulolítica. Neste estudo, objetivouse quantificar e identificar as populações de protozoários no rúmen de novilhos, criados exclusivamente em pastagem, durante o período seco do ano, no Norte de Minas Gerais. Amostras do suco ruminal de 36 novilhos mestiços Nelore foram coletadas diretamente do rúmen, após o abate, durante o inicio, meio ou fim do período de estiagem. Para conservação dos protozoários, $1 \mathrm{~mL}$ do suco ruminal foi diluído em $9 \mathrm{~mL}$ de solução de formaldeido a $10 \%$. Posteriormente, foram realizadas diluições decimais em solução salina e contagem de protozoários pequenos, médios e grandes em câmara de Sedgewick Rafter. As concentrações de protozoários

foram transformadas em $\log _{10}(\mathrm{x}+1)$ e submetidas à análise de variância e as médias comparadas com teste de Duncan. As frequências dos gêneros foram comparadas com o teste do qui-quadrado. As médias da concentração total e de pequenos e médios protozoários diminuíram significativamente com o decorrer do período seco; entretanto, os grandes protozoários não apresentaram alterações na concentração média durante os três períodos avaliados. Foram identificados 7749 protozoários, pertencentes a 17 gêneros diferentes. Observou-se que os gêneros Charonina e Entodinium apresentaram alta ocorrência no início, meio e final do período da seca $(\mathrm{p}<0,05)$, indicando a participação desses ciliados em bovinos alimentados exclusivamente a pasto, durante esse período

do

ano.
\end{abstract}

PALAVRAS-CHAVE: Brachiaria spp., ciliados, microbiota ruminal, pecuária de corte, semiárido.

\section{RUMEN PROTOZOA OF STEERS RAISED ON TROPICAL PASTURE DURING DRY SEASON}

\section{ABSTRACT}

Protozoa of the rumen can represent $40 \%$ of total nitrogen and $60 \%$ of the final product of fermentation and it has cellulolytic activity. This study aimed to quantify and identify the ruminal protozoa populations from steers, raised exclusively on pastures during the dry season in the north of Minas Gerais, Brazil. Samples of ruminal fluid of
36 Nellore steers were collected directly from the rumen immediately after slaughter, during the early, middle or end of the dry season. For protozoa conservation, $1 \mathrm{~mL}$ of ruminal fluid was diluted in $9 \mathrm{~mL}$ of $10 \%$ formaldehydes solution. Decimal dilutions were made in saline and the counting of small, medium and large protozoa was 
performed in Sedgewick Rafter chamber. The protozoa concentrations were transformed into $\log _{10}(\mathrm{x}+1)$, subjected to variance analysis and the means were compared with Duncan test. The genus frequencies were compared with chi-square test. The concentration average of the total and of small and medium protozoa declined significantly over the course of the dry season; however, alterations in large protozoa concentrations were not detected over the three periods evaluated. A total of 7749 protozoa were identified, belonging to 17 different genera. Charonina spp. and Entodinium spp. had the highest occurrence during the three periods of dry season ( $\mathrm{P}<0.05)$, suggesting the participation of these ciliates in rumen cattle fed exclusively on tropical pasture during this

KEYWORDS: Cattle, nutrition, protozoa, ruminal juice, semiarid.

\section{INTRODUÇÃO}

Os ruminantes alimentam-se basicamente de alimentos fibrosos. Ao longo do tempo, esses animais desenvolveram grande capacidade ingestiva, acompanhada por simbiose com microrganismos que digerem a fibra vegetal. Dessa forma, eles obtêm energia para o crescimento, a produção e a reprodução ${ }^{1}$. Esses animais são dependentes da fermentação dos componentes dos alimentos por microrganismos do rúmen, que desempenham funções bioquímicas e fisiológicas importantes, permitindo maior aproveitamento de carboidratos estruturais. A notável capacidade de produção proteica nos ruminantes tem sido atribuída ao sistema de préestômagos, que alberga esse complexo ecossistema microbiano ${ }^{1}$.

A evolução do trato gastrintestinal nos ruminantes proporcionou o desenvolvimento de uma relação simbiótica e mutualística com protozoários ciliados que, junto às bactérias e aos fungos, compõem a microbiota ruminal $^{2}$. Os ciliados possuem considerável atividade celulolítica e fermentativa, são os maiores microrganismos presentes no rúmen, constituindo importante fração da comunidade no ecossistema ruminal. Foram os primeiros a serem descritos nesse nicho e podem representar $40 \%$ do nitrogênio total e $60 \%$ do produto final da fermentação ${ }^{3}$.

Em regiões de clima semiárido, como o Norte de Minas Gerais, a produção de forrageiras de boa qualidade torna-se difícil ao longo de todo o ano por causa do maior período de estiagem. A disponibilidade dos alimentos é comprometida, assim como a qualidade das forrageiras persistentes à seca, que apresentam redução da digestibilidade devido ao processo fisiológico de lignificação da parede celular ${ }^{4}$.

Muitos pesquisadores têm caracterizado a microbiota ruminal e registrado a importante participação dos protozoários na digestão e equilíbrio do ecossistema ruminal e na saúde dos ruminantes; entretanto, poucos estudos avaliam a ecologia e a importância desses microrganismos para animais criados em pastagens tropicais com baixo valor nutricional e frequentemente lignificadas.

Tendo em vista as características das pastagens no período da seca, em regiões semiaridas, é de grande relevância verificar os efeitos desse alimento sobre a população de protozoários que muitas vezes podem retratar as condições do ecossistema ruminal ${ }^{5}$. Nesta pesquisa, avaliou-se o perfil da população de protozoários do rúmen de novilhos Nelore alimentados em pastagens tropicais ao longo do período da seca no Norte de Minas Gerais.

\section{MATERIAL E MÉTODOS}

$\mathrm{O}$ experimento foi realizado com animais advindos de propriedades da zona rural da cidade de Montes Claros, MG. As análises foram realizadas no laboratório de microscopia do Instituto de Ciências Agrárias da Universidade Federal de Minas Gerais, Campus regional de Montes Claros. Montes Claros encontra-se na altitude de $648 \mathrm{~m}$ coodernadas 16\%2'16" de latitude sul e 43\%49'13" de longitude oeste. O clima da região é do tipo $\mathrm{AW}$, segundo a classificação de Köppen, que se caracteriza por uma estação chuvosa no verão e seca no inverno, com vegetação típica de cerrado. A temperatura média anual é de $24,2{ }^{\circ} \mathrm{C}$, a pluviosidade média anual é de aproximadamente $1.050 \mathrm{~mm}$ e o período de estiagem vai de abril a outubro ${ }^{6}$.

A amostragem deste estudo foi constituída por 36 novilhos mestiços Nelore. A análise das arcadas dentárias indicou que apresentavam de 24 a 40 meses de idade. Os animais eram criados exclusivamente sob o sistema extensivo em pastagem de Brachiaria decumbes e suplementados com mistura mineral para gado de corte (Tabela 1). 
Tabela 1. Níveis de garantia por kg do núcleo mineral utilizado no período da seca

\begin{tabular}{lc}
\hline Minerais & Período das secas \\
\hline Cálcio (g/mín-máx*) & $80-100$ \\
Fósforo (g/mín) & 50 \\
Sódio (g/mín) & 65 \\
Magnésio (g/mín) & 8 \\
Enxofre (g/mín) & 25 \\
Flúor (mg/máx) & 500 \\
Zinco (mg/mín) & 2640 \\
Cobre (mg/mín) & 820 \\
Manganês (mg/mín) & 660 \\
Cobalto (mg/mín) & 82 \\
Iodo (g/mín) & 45 \\
Selênio (g/mín) & 18 \\
Nitrogênio não protéico, equivalente PB (g) & 860 \\
\hline
\end{tabular}

*mín = mínimo e máx=máximo. ${ }^{1}$ Composição química segundo o fabricante.

Coletaram-se 11 amostras de fluido ruminal no mês de abril, início do período da seca, 14 no mês de julho, que corresponde ao meio do período, e 11 no final do mês de outubro, correspondendo ao final do período de estiagem. A pluviosidade média em milímetros foi de 16,4 no início, 0,0 no meio e 1,6 milímetros no final do período de seca.

A coleta do fluido ruminal foi realizada em abatedouro com inspeção municipal do Distrito de Montes Claros. Após jejum de 12 a 18 horas, os animais foram abatidos por concussão cerebral e sangria. As amostras foram obtidas imediatamente após o abate, por incisão do saco ventral do rúmen e coleta de aproximadamente $20 \mathrm{~mL}$ de fluido, com auxílio de pipetas estéreis. Todos os procedimentos realizados foram submetidos e aprovados pelo comitê de ética em experimentação animal da UFMG pelo protocolo $\mathrm{n}^{\circ} 156 / 05$.

As amostras foram transportadas em caixas isotérmicas e armazenadas por no máximo uma hora em tubos de ensaio vedados e estéreis. A análise macroscópica do líquido coletado foi realizada imediatamente após a coleta, em tubos de ensaio contendo $5 \mathrm{~mL}$ do suco amostrado. Foram avaliados cor, odor, viscosidade e tempo de redução do azul de metileno. $\mathrm{O} \mathrm{pH}$ foi mensurado utilizando-se um potenciômetro digital ${ }^{5}$.

Posteriormente, amostras de $1 \mathrm{~mL}$ do suco ruminal de cada animal foram diluídas em $9 \mathrm{~mL}$ de solução de formaldeído a 10\% para conservação das estruturas morfológicas dos protozoários ${ }^{7}$. Para a quantificação dos protozoários foram realizadas diluições decimais de $1 \mathrm{~mL}$ da solução de formaldeido em tubos, contendo $9 \mathrm{~mL}$ de solução salina e, em seguida, foram contados os protozoários pequenos, médios e grandes em câmara de Sedgewick Rafter em microscópio óptico ${ }^{8}$.

Para identificação desses microrganismos ruminais, foi utilizada uma gota da diluição $10^{-1}$ ou $10^{-2}$, como descrita anteriormente, juntamente com uma gota de lugol em lâminas de microscopia. Nessas lâminas, foram acopladas lamínulas para visualização, em microscópio óptico, utilizando-se objetiva de 40X, das microestruturas dos protozoários e posterior classificação de acordo com a chave descrita em Dehority?

$\mathrm{O}$ delineamento do experimento foi inteiramente casualizado. As repetições foram representadas por 11,14 e 11 novilhos coletados respectivamente no início, meio e final do período da seca. Após análise exploratória (teste de Liliefors e teste de Cochran e Bartlett), as concentrações de protozoários obtidas foram transformadas em $\log _{10}$ $(\mathrm{x}+1)$ e submetidas à análise de variância. As médias foram comparadas utilizando-se teste de Duncan. Para análise das ocorrências dos gêneros foi utilizado o teste do qui-quadrado. Os dados foram processados no sistema para análises estatísticas e genéticas (SAEG) (9), considerando-se o nível de significância de $5 \%$.

\section{RESULTADOS E DISCUSSÃO}

$\mathrm{O}$ pH do fluido ruminal não apresentou diferença significativa entre os três períodos de coleta, com valor médio de 6,8 . Uma vez que a dieta era composta exclusivamente por forragem. Os valores de $\mathrm{pH}$ encontrados apresentaram-se normais, já que dietas ricas em fibras proporcionam maior tempo de ruminação e, consequentemente, maior 
produção de saliva, elevando o $\mathrm{pH}$; entretanto, o jejum de 12 horas antes do abate poderia proporcionar um pequeno aumento no $\mathrm{pH}$ ruminal ${ }^{7}$ ${ }^{10}$. Segundo Dirksen ${ }^{5}$, o valor normal do $\mathrm{pH}$ do conteúdo ruminal oscila entre 5,5 e 7,4 ao longo do dia, de acordo com a alimentação administrada e com o intervalo de tempo da última alimentação.

Para características de cor do líquido ruminal, pôde-se observar que todas as amostras apresentaram coloração castanha-esverdeada. Dirksen $^{5}$ relata que a cor do suco ruminal é estabelecida de acordo com a alimentação ofertada ao animal e animais a pasto apresentam fluido ruminal com coloração verde oliva ou castanhoesverdeado, como a observada neste estudo.

$\mathrm{O}$ odor do fluído ruminal foi aromático para ambas as amostras. A viscosidade foi levemente espessa e a redução do azul de metileno apresentouse superior a seis minutos para $83,3 \%$ dos animais avaliados e, até seis minutos, para os demais. Dirksen $^{5}$ relatou que animais com alimentação constituída com grande quantidade de fibra apresentam redução do azul de metileno entre três e seis minutos. Por outro lado, prolonga-se para até mais de 15 minutos na inatividade simples da microbiota em animais alimentados com dietas pobres em energia e proteína ou em bovinos com inapetência prolongada. Neste estudo, os valores elevados para essa característica em 30 amostras sugerem uma microbiota pouco ativa, principalmente para o meio e final do período da seca, quando as pastagens, frequentemente, possuem qualidade inferior.

A quantidade total de protozoários diminuiu significativamente com a evolução do período de seca $(p<0,05)$. As concentrações de protozoários pequenos, médios e grandes, avaliadas neste estudo, encontram-se dispostas na Tabela 2. A partir dos resultados obtidos, pôde-se observar que a média de pequenos protozoários foi maior durante todo o período da seca, seguida da média de médios e, posteriormente, de grandes ciliados $(\mathrm{p}<0,05)$.

Tabela 2 - Efeito do período da seca sobre a contagem média de protozoários por $\mathrm{mL}$ de suco ruminal e coeficiente de variação (\%) para novilhos criados no norte de Minas Gerais, Brasil

\begin{tabular}{lllll}
\hline & Período da Seca & Meio & Fim & CV $(\%)$ \\
Protozoários & Início & $4,5 \times 10^{4} \mathrm{Ab}$ & $1,8 \times 10^{4} \mathrm{Ac}$ & 3.89 \\
\hline Pequenos & $7,8 \times 10^{4} \mathrm{Aa}$ & $1,0 \times 10^{4} \mathrm{Bb}$ & $7,2 \times 10^{3} \mathrm{Bc}$ & 3.36 \\
Médios & $4,8 \times 10^{4} \mathrm{Ba}$ & $2,4 \times 10^{3} \mathrm{Ca}$ & $2,3 \times 10^{3} \mathrm{Ca}$ & 6.31 \\
Grandes & $2,6 \times 10^{3} \mathrm{Ca}$ & $5,8 \times 10^{4} \mathrm{~b}$ & $2,7 \times 10^{4} \mathrm{c}$ & 2.96 \\
Total & $1,3 \times 10^{5} \mathrm{a}$ & 4,25 & 3,71 & \\
CV $(\%)$ & 3.62 & & \\
\hline
\end{tabular}

Médias seguidas de mesma, letra minúscula na linha e maiúsculas nas colunas, não diferem entre si, ao nível de 5\% pelo teste de Duncan.

Os dados indicaram que o início da seca proporciona condições mais favoráveis para o desenvolvimento de pequenos e médios protozoários, comparando-se com o restante da estação. Entretanto, os grandes protozoários foram encontrados na mesma proporção no início, meio e final da seca $(p>0,05)$. A concentração total de protozoários encontrados está dentro dos valores normais já reportados que correspondem a $10^{4}$ a $10^{6}$ protozoários/mL de conteúdo ruminal ${ }^{11-12}$.

Evidenciou-se nesta pesquisa que o grupo dos grandes protozoários se manteve estável durante todo o período da seca, sugerindo a importância da ocorrência de protozoários pertencentes a esse grupo em animais criados a pasto, no Norte de Minas Gerais, onde as gramíneas utilizadas são do tipo C4. As pastagens em que os animais se encontravam eram constituídas principalmente por Brachiaria decumbens. Essa gramínea, durante a seca, tem apresentado valores de matéria seca (MS), proteína bruta (PB), fibra em detergente neutro (FDN) e fibra em detergente ácido (FDA) de 46,9; 6,3; 78,3 e $45,1 \%$, respectivamente ${ }^{13}$. É possível pressupor que, em regiões com alta luminosidade e baixa pluviosidade, gramíneas C4 podem apresentar grande produção de carboidratos estruturais, expressando, assim, valores superiores a $35,7 \%$ de $(\mathrm{MS})^{14}$.

No estudo de Natsuki et al. ${ }^{15}$, foi verificado que a proporção de parede celular vegetal primária em relação à parede celular vegetal secundária em materiais fecais foi menor para os defaunados do que para os bovinos com protozoários. Os autores indicaram que a defaunação aumentou a degradação microbiana das paredes celulares finas, mas reduziu a degradação das paredes celulares desenvolvidas, indicando a importância desses protozoários no rúmen de animais criados a pasto com forragens em estádio fenológico avançado.

A partir dos resultados desta pesquisa, pôde- 
se inferir que a maturidade da forrageira ao longo do período seco influenciou o decréscismo da populaçao de protozoarios totais. Esse resultado corrobora o estudo de Nogueira Filho et al. ${ }^{16}$, que constatou que o estádio fenológico avançado do capim elefante influenciou negativamente a concentração de protozoários do rúmen. Veira ${ }^{17}$ e Grubb e Dehority ${ }^{18}$ afirmaram que o número de protozoários pode ser relativamente baixo em animais recebendo dietas exclusivas de forragens ou com maior proporção de forragens em relação ao concentrado.

Estudos têm comprovado a presença de atividades hemicelulolítica e celulolítica nos ciliados do rúmen, especialmente do grupo dos grandes entodiniomorfos ${ }^{19}$. Segundo Orpin e Letcher $^{20}$, os grandes protozoários ciliados colonizam fragmentos da fibra, ingerem diretamente os tecidos da planta e facilitam a ação de bactérias específicas. Dessa forma, pode-se justificar a importância de grandes protozoários em bovinos criados em pastagem tropical durante o período seco, pois essa atividade degradativa da parede celular vegetal acarreta melhor aproveitamento das forragens que geralmente são de baixa qualidade nesse período.

A distribuição dos gêneros de protozoários identificados nesta pesquisa encontra-se na Tabela 3. Os gêneros Charonina e Entodinium foram os de maior ocorrência no início, meio e fim da seca $(p<0,05)$. Foram identificados 17 gêneros, encontrados nos três períodos da seca, ou seja, a diversidade da população de protozoários foi semelhante ao longo de todo o período seco. Ciliados das famílias Blepharocorythidae (Charonina spp.), Isotrichidae (Isotricha spp.) e Ophryoscolecidae (Entodinium spp., Eodinium spp. e Diplodinium spp) apresentaram alta ocorrência no rúmen desses animais alimentados em pastagem de Brachiaria spp..

Corroborando esse dado, Dehority e Odenyo $^{21}$, ao compararem ruminantes africanos selvagens e domésticos, observaram maior diversidade das populações de protozoários ciliados em animais mantidos exclusivamente sob pastejo, em relação a outros que eram alimentados com concentrado.

Tabela 3 - Distribuição dos gêneros de protozoários ruminais provenientes de novilhos Nelore criados a pasto no período da seca no norte de Minas Gerais, Brasil

\begin{tabular}{|c|c|c|c|c|}
\hline \multirow[b]{2}{*}{ Gêneros } & \multicolumn{4}{|l|}{ Período da seca } \\
\hline & Início & Meio & Final & Totais \\
\hline Buetschilia spp. & $111(4,2 \%) \mathrm{a}$ & $191(5,9 \%) b$ & $181(9,7 \%) \mathrm{c}$ & 483 \\
\hline Isotricha spp. & $253(9,5 \%)$ a & $248(7,7 \%) b$ & $232(12,5 \%) \mathrm{c}$ & $733 \mathrm{C}$ \\
\hline Dasytricha spp. & $132(4,9 \%) \mathrm{a}$ & $209(6,5 \%) b$ & $102(5,5 \%) \mathrm{a}$ & 443 \\
\hline Charonina spp. & $716(26,9 \%)$ a & $698(21,6 \%) b$ & $435(23,4 \%) b$ & $1849 \mathrm{~A}$ \\
\hline Entodinium spp. & $485(18,2 \%)$ a & $452(13,9 \%) b$ & $366(19,7 \%)$ a & $1303 \mathrm{~B}$ \\
\hline Diplodinium spp. & $155(5,8 \%) \mathrm{a}$ & $313(9,7 \%) b$ & $177(9,5 \%) b$ & $645 \mathrm{D}$ \\
\hline Eodinium spp. & $280(10,5 \%)$ a & $318(9,9 \%)$ a & $145(7,8 \%) b$ & $743 \mathrm{C}$ \\
\hline Eremoplastron spp. & $195(7,3 \%) \mathrm{a}$ & $192(5,9 \%) b$ & $87(4,7 \%) b$ & 474 \\
\hline Eudiplodinium spp. & $41(1,5 \%) \mathrm{a}$ & $188(5,8 \%) b$ & $32(1,7 \%)$ a & 261 \\
\hline Diploplastron spp. & $62(2,3 \%) \mathrm{a}$ & $135(4,2 \%) \mathrm{b}$ & $35(1,9 \%)$ a & 232 \\
\hline Polyplastron spp. & $22(0,8 \%)$ a & $56(1,7 \%) \mathrm{b}$ & $16(0,9 \%)$ a & 94 \\
\hline Ostracodinium spp. & $49(1,8 \%)$ a & $116(3,6 \%) b$ & $19(1 \%) \mathrm{c}$ & 184 \\
\hline Elytroplastron spp. & $12(0,5 \%) \mathrm{a}$ & $14(0,4 \%) \mathrm{a}$ & $9(0,5 \%) \mathrm{a}$ & 35 \\
\hline Metadinium spp. & $3(0,1 \%)$ a & $15(0,5 \%) b$ & $12(0,6 \%) b$ & 30 \\
\hline Enoploplastron spp. & $33(1,2 \%)$ a & $7(0,2 \%) b$ & $5(0,3 \%) b$ & 45 \\
\hline Ophyriscilex spp. & $19(0,7 \%) \mathrm{a}$ & $7(0,2 \%) b$ & $2(0,1 \%) b$ & 28 \\
\hline Epidinium spp. & $91(3,4 \%) \mathrm{a}$ & $70(2,2 \%) b$ & $6(0,3 \%) c$ & 167 \\
\hline Total & $2659(100 \%)$ & $3229(100 \%)$ & $1861(100 \%)$ & 7749 \\
\hline
\end{tabular}

Frequências com mesmas letras, maiúsculas na coluna e minúsculas nas linhas, não diferem entre si, comparadas estatisticamente pelo teste de qui-quadrado a $5 \%$ de probabilidade.

Franzolim e Franzolim $^{19}$ descrevem a presença dos gêneros Entodinium, Isotricha e Dasytricha no rúmen de bovinos de corte alimentados com cana-de-açúcar picada e com suplementação com concentrado. No estudo realizado por esses autores, as espécies do gênero Entodinium compreenderam à grande maioria da composição de protozoários ruminais de bovinos 
$(79,2 \%)$. Esse gênero foi o mais frequente no rúmen de bovinos arraçoados com três dietas à base de capim elefante e concentrado, constituindo de $75 \%$ a $79,2 \%$ do total de ciliados em animais recebendo dietas compostas por capim-elefante com taxas de inclusão de concentrado de até $40 \%^{22}$. Esse protozoário também foi o mais frequente entre os ciliados de bovinos zebuínos e bubalinos alimentados com feno Cynodon dactylon e concentrado $^{23}$.

Os gêneros Epidinium e Ophryoscolex são conhecidos pela relação de antagonismo estabelecida entre ambos, de forma que, frequentemente, não estabelecem populações mistas ${ }^{24}$; entretanto, neste estudo, esses dois gêneros foram observados em coexistência para os três periodos avaliados, constituindo populações mistas de protozoarios.

Martinele et al. $^{25}$ evidenciaram que os gêneros Entodinium, Isotricha e Charonina foram os mais frequentes para bovinos alimentados com capim-elefante. Nesta pesquisa, além desses três gêneros, verificou-se também a alta incidência do gênero Eodinium, como pode ser observado na Tabela 2.

A dieta, provavelmente, é um dos fatores mais importantes para a concentração e a distribuição dos gêneros de protozoários existentes no rúmen ${ }^{26}$. Nesta pesquisa, os resultados verificados para o $\mathrm{pH}$, cor, odor e viscosidade do fluido ruminal demonstram que, aparentemente, o principal efeito de variações estacionais sobre os protozoários ciliados esteja relacionado à fenologia da forragem ${ }^{27}$, 16 .

Franzolim et al. ${ }^{28}$ observaram que dietas com níveis crescentes de fibra em detergente neutro (FDN) reduzem a população de ciliados no conteúdo ruminal dos bovinos de forma mais significativa que a de bubalinos, apesar de haver redução do número total e de quase todos os gêneros de protozoários ciliados com nível alto de FDN para ambos os ruminantes.

Rufino et al. ${ }^{29}$ indicaram que a iclusão de $10 \%$ de torta de macaúba, que apresenta alto teor de FDN, na dieta de ruminantes ocasionou maior ocorrência de protozoários do gênero Charonina, que também apresentou alta frequencia nos três períodos de seca avaliados neste estudo.

Nogueira Filho et al. ${ }^{16}$ avaliaram os efeitos do fornecimento de forragem em diferentes estádios de crescimento vegetativo sobre os protozoários ciliados no rúmen de bovinos, verificando que o número total de ciliados, bem como os dos gêneros Entodinium, Diplodinium e Polyplastron, diminuíram com o avançar da idade da planta forrageira. Esses autores atribuíram esta queda no número de protozoários à diminuição de açúcares solúveis e ao aumento da fibra, conforme a maturação da planta.

Durante a estação seca, os representantes do gênero Epidinium apresentaram decréscimo na ocorrência, embora sejam citados por Manella e Lourenço ${ }^{27}$ e Williams e Coleman ${ }^{91}$ por participarem ativamente na degradação de material rico em celulose. Os resultados deste estudo corroboram os relatos de Bonhomme-Florentin et $\mathrm{al}^{30}$, que avaliaram os efeitos da sazonalidade sobre as populações de ciliados de bovinos e verificaram que houve significativo decréscimo no número e nas espécies de ciliados do gênero Epidinium durante a estação seca.

\section{CONCLUSÕES}

A população de pequenos e médios protozoários do rúmem de novilhos criados em região semiárida diminui com a evolução do período da seca; entretanto, a concentração média dos grandes protozoários é constante ao longo desse período. Os gêneros Charonina e Entodinium são os mais frequentes no início, meio e final da seca.

\section{AGRADECIMENTOS}

Este trabalho teve apoio da Capes (Coordenação de Aperfeiçoamento de pessoal de Nível Superior), do Conselho Nacional de Desenvolvimento Científico e Tecnológico (CNPq), da Fundação de Amparo à Pesquisa do Estado de Minas Gerais (FAPEMIG) e da Pró-reitoria de Pesquisa da Universidade Federal de Minas Gerais.

\section{REFERÊNCIAS}

1. Willians, AG; Coleman, GS The rumen protozoa. Springer-Verlag, New York Inc; 1991. p. 423.

2. Mackie, RI Mutualistic fermentative digestion in the gastrointestinal tract: diversity and evolution. Integrative and Comparative Biology. 2002;42:319-326.

3. Ryle, M, Ørskov, ER Ciliados de la panza y piensos tropicales. Revista Mundial de Zootecnia. 1987;64:21-30.

4. Carvalho, GGP; Pires, AJV Organização dos Tecidos de Plantas Forrageiras e suas Implicações para os Ruminantes. Archivos de Zootecnia. 2008;57(R):13-28.

5. Dirksen, G Sistema digestivo. In: Dirksen,G; Grunder,HD.; Stober, M (Ed.). Rosenberger: Exame Clínico dos Bovinos. Rio de Janeiro: Guanabara-Koogan; 1993. p.167-169. Portuguese.

6. INMET - Instituto Nacional de Meteorologia [Internet]. 2010 Nov 01 [citado 2010 Nov 16]. Disponivel em:http://www.inmet.gov.br/projetos/rede/pesquisa/gera_s 
erie txt_mensal.php?\&mRelEstacao $=83437 \&$ btnProcesso $=$ serie $\& m$ RelDtInicio $=01 / 04 / 2010 \& m R e l D t F i m=01 / 10 / 20$ $10 \& \mathrm{mAtributos}=, \ldots, \ldots, \ldots, 1,1,1,1$. Portuguese.

7. Dehority, BA Laboratory manual for classification and morphology of rumen ciliate protozoa. Florida: CRC Press Inc; 1993. p. 96.

8. Dehority, BA. Evaluation of subsampling and fixation procedures used for counting rumen protozoa. Applied and Environmental Microbiology. 1984,48:182-185.

9. SAEG Sistema para Análises Estatísticas - SAEG. Versão 9.1: Fundação Arthur Bernardes. Viçosa: UFV., 2007.

10. Matos, DS; Guim, A; Batista, AMV, Santos, MVF, Correa, IM, Santos, GRA, Lopes, CRA. População de protozoários ciliados no rúmen de ovinos criados na caatinga de Pernambuco. Revista Brasileira de Saúde e Produção animal. 2008;9:270-279.

11. Dehority, BA. Rúmen microbiology. Wooster: ohio state university; 1987. 239p.

12. Kamra, DN. Rumen microbial ecosystem. Current Science. 2005;89:124-135.

13. Cavalcanti Filho, LFM, Santos, MVF, Ferreira, MA. Lira, MA, Modesto EC, Dubeux Jr. JCB, Ferreira, RLC Silva, MJ. Caracterização de pastagem de Brachiaria decumbens na zona da mata de Pernambuco. Archivos de Zootecnia.2008;57:391-402.

14. Paciullo, DSC.; Gomide, JAL., Silva, EAM., Queiroz, DS.; Gomide, CAM. Características anatômicas e nutricionais de lâminas coliares e colmos de gramíneas forrageiras, em função do nível de inserção no perfilho, da idade e da estação de crescimento. Revista Brasileira de Zootecnia. 2002;31:890-899.

15. Natsuki, K.; Itsuko, W.; Mimune, S.; Hirochi, K.; Hisao, I. Effect of presence of rumen protozoa on degradation of cell wall constituents in gastrointestinal tract of cattle. Animal Science Journal. 2007;78:275-280.

16. Nogueira Filho, JCM, Lucci, CS, Melotti, L, Oliveira, MEM, Lima,GC, Cunha, JA. Contagens diferenciais de protozoários ciliados em rúmen de bovinos arraçoados com capim elefante Napier (Pennisetum purpureum Schum), em vários estádios de crescimento vegetativo. Brazilian Journal of Veterinary Research and Animal Science. 1992;27:215-221.

17. Veira, DM. The role of ciliate protozoa in nutrition of the ruminant. Journal Animal Science. 1986;63:15471560 .

18. Grubb, JA, Dehority, B. Effects of an abrupt change in ration from all roughage to high concentrate upon rumen microbial number in sheep. Journal of Applied Microbiology. 1975,30:402-412.

19. Franzolin, R., Franzolin, MHT. População de protozoários ciliados e degradabilidade ruminal em búfalos e bovinos zebuínos sob dieta à base de cana de açúcar. Revista Brasileira de Zootecnia. 2000,29:18531861.

20. Orpin, CG, Letcher, AJ. Some factors controlling the attachment of the rumen holotrich protozoa Isotricha intestinalis and I. prostoma to plant particles in vitro. Journal of General Microbiology. 1978,106(5):33-40.

21. Dehority, BA, Odenyo, AA, Influence of diet on the rúmen protozoal fauna of indigenous african wild ruminants. The Journal of Eukaryotic Microbiology. 2003,50:220-223.

22. Martinele, I, Santos, GRA, Matos, DS, Batista, AVB, D'Agosto, M. Protozoários ciliados do rúmen de ovinos mestiços mantidos em pastagem natural de caatinga. Revista Brasileira de Saúde e Produção animal. 2008,9:280-292.

23. Nogueira Filho, JCM, Oliveira, MEM, Cunha, JA, Toledo, LR. Volume líquido e taxa de turnover no rúmen de zebuínos e bubalinos submetidos a dietas com volumosos e concentrados e sua relação com protozoários ciliados. Ciência Animal Brasileira. 2004,5:1-7.

24. Göçmen, B, Dehority, BA, Talu, GH, Rastgeldy, S. The rumen ciliate fauna of domestic sheep (Ovis ammon aires) from the Turkish Republic of Northern Cyprus. The Journal of Eukaryotic Microbiology. 2001,48:455-459.

25. Martinele, I, Detoni, ML, Rust, NM, D’Agosto, M. Protozoários ciliados (Protista, Ciliophora) no conteúdo do rúmen e do retículo de bovinos. Revista Brasileira de Zoociências. 2007,9:63-67,

26. Williams, AG. Rumen holotrich ciliate protozoa. Microbiological Reviews. 1986,50:25-49.

27. Manella, MQ, Lourenço, AJ. População de protozoários ciliados no rúmen de bovinos nelore em pastos de Brachiaria brizantha Marandu recebendo suplemento proteico ou com livre acesso a banco de proteína de Leucaena leucocephala nas diferentes estações do ano. Boletim de Indústria Animal. 2004,61:01-11.

28. Franzolin, MHT, Silveira, AC, Franzolin, R. Effects of diets with different levels of neutral detergent fiber and the porous size of nylon bags incubated in the rumen on ruminal fauna in buffaloes and cattle. Revista Brasileira de Zootecnia. 2002,31:716-723.

29. Rufino, LMA, Barreto, SMP., Duarte, ER., Geraseev, L.C. Efeitos da inclusão de torta de macaúba sobre a população de protozoários ruminais de caprinos. Revista Brasileira de Zootecnia. 2011,40:899-903.

30. Bonhomme-Florentin, A, Blancou, J, Latteur, B, E'tude des variations saisonnières de la microfaune du rumen de zebus. Protistologica. 1978,14:283-289. 